\title{
The implementation of a smoking cessation and alcohol abstinence intervention for people experiencing homelessness
}

\section{Rebekah Pratt ( $\square$ rjpratt@umn.edu )}

University of Minnesota System https://orcid.org/0000-0003-3561-8276

\section{Serena Xiong}

University of Minnesota

Azul Kmiecik

University of Minnesota

Cathy Strobel-Ayres

University of Minnesota

Anne Joseph

University of Minnesota

Susan A Everson-Rose

University of Minnesota

Xianghua Luo

University of Minnesota

Ned Cooney

Yale University School of Medicine

Janet Thomas

University of Minnesota

Shelia Specker

University of Minnesota

Kola Okuyemi

University of Utah

\section{Research}

Keywords: CFIR, PTQ2, CFIR, implementation process, characteristics of individuals

Posted Date: December 28th, 2020

DOI: https://doi.org/10.21203/rs.3.rs-133182/v1 
License: (c) (i) This work is licensed under a Creative Commons Attribution 4.0 International License. Read Full License 


\section{Abstract}

In the United States, eighty percent of the adult homeless population smokes cigarettes compared to 15 percent of the general population. In 2017 Power to Quit 2 (PTQ2), a randomized clinical trial, was implemented in two urban homeless shelters in the Upper Midwest to address concurrent smoking cessation and alcohol treatment among people experiencing homelessness. A subset of this study population were interviewed to assess their experiences of study intervention. The objective of this study was to use participants' experiences with the intervention to inform future implementation efforts of combined smoking cessation and alcohol abstinence interventions, guided by the Consolidated Framework for Implementation Research (CFIR).

Methods: Qualitative semi-structured interviews were conducted with 40 PTQ2 participants between 2016-2017 and analyzed in 2019. Interviews were audio-recorded, transcribed, and analyzed using a socially constructivist approach to grounded theory.

Findings: Participants described the PTQ2 intervention in positive terms. Participants valued the opportunity to obtain both counseling and nicotine-replacement therapy products (intervention characteristics) and described forming a bond with the PTQ2 staff and reliance on them for emotional support and encouragement (characteristics of individuals). However, the culture of alcohol use and cigarette smoking around the shelter environment presented a serious challenge (outer setting). The study setting and the multiple competing needs of participants were reported as the most challenging barriers to implementation (implementation process).

Conclusion: The CFIR was useful in offering specific insights about the implementation context of the PTQ2 intervention, including the impact of the factors in the inner and outer settings of the shelter environment. Participants described a discord in their personal goals of reduction compared with the study goals of complete abstinence, which may pose a challenge to the ways in which success is defined for people experiencing homelessness.

\section{Contributions To The Literature}

- People experiencing homelessness have worryingly high rates of smoking, but do express motivation to quit smoking. However, intervention adaptation is required to effectively address this population's unique needs.

- This study addresses the lack of research on the implementation of smoking cessation interventions that target people experiencing homelessness, despite the mixed success of such programs on cessation outcomes.

- To our knowledge, this is the first time that CFIR has been applied to the implementation of a smoking and alcohol cessation intervention for smokers experiencing homelessness.

\section{Introduction}


Approximately $1.5 \%$ of adults living in the United States experience homelessness annually and up to $4.2 \%$ of adults living in the United States will experience homelessness in their lifetime.(1) Homelessness presents a unique set of challenges that can negatively impact health(2) and presents an important public health concern. Eighty percent of the adult homeless population smokes cigarettes(3) compared to 15 percent of the general population(4), therefore determining ways to engage this community in smoking cessation is crucial to mitigating the impact of homelessness on preventable mortality and morbidity(4). While smokers experiencing homelessness report interest in smoking cessation (5-8), there are multiple competing priorities and barriers demands, in particular, concerns about the social environment and daily stress. $(7,9-11)$ Cessation intervention efforts to date have resulted in minimal quit rates. $(4,12)$

There is a paucity of information on the processes involved in implementing smoking cessation interventions among people experiencing homelessness. Implementation Science (IS) studies the process of intervention implementation(13) and may offer a valuable perspective in better understanding how cessation approaches could best be implemented for people experiencing homelessness. The Consolidated Framework for Implementation Research (CFIR)(14-16) has been widely used in health services research(15) and focuses on five key areas of implementation.

The first CFIR domain focuses on the intervention characteristics, including the perceived strength and quality of the intervention, the relative advantage and adaptability of the intervention and the source of the intervention content.(14) The second domain is termed the inner setting, which considers culture and climate and the fit between individual participant values and the intervention content.(14) The third domain is the outer setting, which focuses on patient needs and resources, peer pressure and the broader policy context in which the intervention is delivered.(14) The fourth domain pertains to the characteristics of the individual participant including self-efficacy, knowledge, beliefs, and readiness to change.(14) Finally, the fifth domain focuses on the implementation process, such as the role of engagement and evaluation.(14) CFIR has been applied to the field of smoking cessation $(17,18)$ and substance use disorders, $(19,20)$ but has not been utilized to understand the unique characteristics of smoking behavior change among those living with homelessness.

Overall, there is little research published that would inform the CFIR domains and smoking cessation for people experiencing homelessness. There is some literature that helps to inform the third domain of the outer setting, in particular the impact of pro-smoking norms commonly found in shelter environments, $(8$, $21,22)$ including high rates of smoking among people frequenting shelters $(6,11)$, making it particularly challenging to quit. $(8,10,21)$ Alternately, stable housing has been positively associated with abstinence outcomes, $(22,23)$ although shelters may offer access to supportive health services $(24)$ to help with addressing smoking.

The majority of the literature published focuses on the fourth CFIR domain, the characteristics of individuals utilizing a smoking cessation intervention, and these have identified psychosocial variables such as shame and stigma around smoking. $(25,26)$ Additionally, there is high prevalence of concurrent 
tobacco and alcohol use among people experiencing homelessness. (27) and it may be beneficial to address these two behaviors simultaneously. $(28,29)$

Studies that have targeted smoking cessation among smokers with alcohol use disorders and findings show an average 7 percent quit rate, and high rates of relapse.(30) Some evidence suggests that addressing smoking can improve alcohol abstinence(31), although studies show mixed results.(32, 33).

Power to Quit 2 (PTQ2) was a randomized controlled trial, built on the findings from the first PTQ study, aimed to investigate concurrent smoking cessation and alcohol treatment among people experiencing homelessness. $(34,35)$ In this study, we present findings from semi-structured interviews with participants completing PTQ2, where the experience of participating in a smoking and alcohol intervention were explored in order to provide insight into the challenges faced by participants when trying to quit smoking. Additionally, the analysis drew on the CFIR framework(14) to inform future learning on the intervention implementation process.

\section{Methods}

PTQ2 was a randomized clinical trial focusing on tobacco and alcohol use that used a three-group design that included (1) Usual care (UC) for smoking and alcohol cessation (control group), (2) Intensive smoking cessation plus UC alcohol abstinence counseling (IS), and (3) Integrated Intensive Smoking and Intensive Alcohol Counseling (IntS + A). The counselling was a cognitive behavioral therapy approach to smoking cessation and alcohol abstinence, and conducted as individual sessions. All participants received 12 weeks of nicotine replacement therapy, with nicotine patches (tailored to their baseline cigarettes smoked per day), plus their choice of nicotine gum or lozenge. A full explanation of the design and methods can be found elsewhere. $(34,35)$ During the RCT consent process, PTQ2 participants were informed that they might be invited to participate in an interview portion of the study. Research study staff approached potential participants just prior to the final study visit (week 26). In recognition of their time and effort, participants were compensated with a $\$ 20$ gift card.

\section{Study Population}

A convenience sample of 40 PTQ2 participants was recruited to take part in the interviews. Interviews were conducted with 25 intervention (IS, IntS + A) and 15 control group participants. The eligibility criteria was that participants had concluded participation in the study intervention or control study conditions, within four weeks of the interview.

\section{Study Instrument}

The research study team developed the semi-structured interview guide with a goal of collecting data on the implementation of the study from the perspective of the participants. The interview guide consisted of 17 questions on the following topics: participants' experience of attempting to quit smoking during the 
study, their experience with the study intervention, and their overall views on participating in research. Interviews lasted between 20 to 60 minutes.

\section{Data Collection}

Semi-structured interviews were conducted in-person between December 2016 and April 2017. In order to avoid bias responses to questions regarding the study and the study team, a Masters in Public Health trained, non-study staff member (AK) conducted the interviews. Interviews were conducted in two of the urban shelters where the study team was delivering the intervention, and were conducted in a private space with the interviewer and interviewee. One interview was conducted with two interviewees together with the interviewer.

\section{Data Analysis}

Interviews were audio recorded, transcribed verbatim and the qualitative data were analyzed in 2019 using NVivo 12.(36) Three members of the research team coded the transcripts (RP, AK and GR), and double coded a sub-set of data to ensure consistency. Training on the analytic process was provided by the lead coder (RP). The research team used the social constructivist approach to grounded theory to identify themes and sub-themes in the data. $(37,38)$ This approach allows for themes to emerge from the data, and be considered in relation to literature or theoretical frameworks, such as CFIR. Discussions with

all members of the research team on the emerging analysis further validated the rigor of the qualitative analysis, with a consensus building approach used to integrate different team member perspectives into the emerging analysis.

\section{Human Subjects}

The University of Minnesota Institutional Review Board provided ethical approval for the conduct of this study.

\section{Findings}

We present participant demographics, followed by key findings from the interviews in relation to overarching CFIR domains (Intervention Characteristics, Outer Setting, Inner Setting, Characteristics of Individuals, Implementation Process).

\section{Demographics}

Baseline demographic characteristics of the subset of participants from the RCT who participated in the interviews are shown in Table 1. Thirty-two participants identified as African American/Black, six as White, one as Native American/Alaska Native, and one as more than one race. Eleven participants were female, and 29 were male. Housing stability was assessed by self-report on a scale of 0 (not at all stable) to 10 (extremely stable) and the mean ( \pm SD) response was $3.53 \pm 3.48$ (range, 0 to 10 ). Most participants identified themselves as unemployed. Participants smoked on average $14.6 \pm 8.3$ (range 2.5 to 40) cigarettes per day at their eligibility screening and just over half had their first cigarette of the day within 
30 minutes of waking. The Alcohol Use Disorder Identification Test (AUDIT),(39) a 10-item scale that measures drinking behavior, dependence, and consequences related to drinking, was used to measure alcohol use severity. Participant AUDIT scores averaged $14.9 \pm 4.87$ (range 7 to 24 ) which corresponds to risky/hazardous or high-risk/harmful alcohol use risk levels. 
Table 1

Participant Baseline Demographics and Characteristics

Mean \pm SD (range)

or $\mathrm{n}(\%)$

N

40

Study randomization arm

A: Standard Care

$15(37.5 \%)$

B: Intensive Smoking Intervention

$13(32.5 \%)$

C: Intensive Smoking and Alcohol Intervention

$12(30.0 \%)$

Age

$50.20 \pm 9.2(29.6-$ 69.5)

Sex

Male

$29(72.5 \%)$

Female

$11(27.5 \%)$

Cigarettes smoked per day (on eligibility survey) ${ }^{1}$

$14.6 \pm 8.3(2.5-40)$

Housing situation (at eligibility survey)

Emergency or overnight shelter

$23(57.5 \%)$

Campsite, vehicle, abandoned building/house, parking garage, or on the street

$7(17.5 \%)$

Transitional or supportive housing, long-term shelter

$5(12.5 \%)$

Staying with relative, friend, or other people/double-up - less than 3 months at

$5(12.5 \%)$

the same place

Housing stability (self-rating from 0 -not at all stable to 10 -extremely stable)

$3.53 \pm 3.48(0-10)$

Race

African American or Black

$32(80.0 \%)$

Native American/Alaskan Native

$1(2.50 \%)$

White

$6(15.0 \%)$

More than 1 race

$1(2.5 \%)$

Education

Some high school or less

$12(30.0 \%)$

${ }^{1} n=4$ participants smoked $<5$ CPD in the 7 days prior to the eligibility survey, but had missing data for their avg. CPD. For these participants, 2.5 CPD was assumed. 


\begin{tabular}{|c|c|}
\hline & $\begin{array}{l}\text { Mean } \pm \text { SD (range) } \\
\text { or } n(\%)\end{array}$ \\
\hline High school graduate or GED & $14(35.0 \%)$ \\
\hline Some college or technical school & $13(32.5 \%)$ \\
\hline Unknown/not reported & $1(2.5 \%)$ \\
\hline \multicolumn{2}{|l|}{ Employment } \\
\hline Employed full time & $2(5.0 \%)$ \\
\hline Employed part time & $4(10.0 \%)$ \\
\hline Out of work for more than 1 year & $8(20.0 \%)$ \\
\hline Out of work for less than 1 year & $7(17.5 \%)$ \\
\hline Unable to work or disabled & $19(47.5 \%)$ \\
\hline \multicolumn{2}{|l|}{ Income } \\
\hline Less than $\$ 400$ per month & $17(42.5 \%)$ \\
\hline$\$ 400-\$ 799$ per month & $15(37.5 \%)$ \\
\hline$\$ 800-\$ 1,199$ per month & $6(15.0 \%)$ \\
\hline$\$ 1,200-\$ 1,799$ per month & $2(5.0 \%)$ \\
\hline Number of children & $2.73 \pm 2.21(0-10)$ \\
\hline MINI Psychotic Symptoms Score at Baseline & $0.58 \pm 1.11(0-4)$ \\
\hline Marijuana use $\geq 20$ days in prior 30 days ( $n, \%$ yes) & $3(7.5 \%)$ \\
\hline Rost-Burnam Screener for Drug Abuse ( $\mathrm{n}, \%$ yes) & $37(92.5 \%)$ \\
\hline Depressive Symptoms (PHQ-9) & $7.38 \pm 6.36(0-23)$ \\
\hline Perceived Stress (PSS-4) & $6.35 \pm 3.05(1-13)$ \\
\hline Anxiety (MINI) & $2.13 \pm 2.95(0-9)$ \\
\hline \multicolumn{2}{|l|}{ FTND Minutes to 1st Cigarette } \\
\hline $0-5$ minutes & $13(32.5 \%)$ \\
\hline $6-15$ minutes & $8(20.0 \%)$ \\
\hline 16-30 minutes & $9(22.5 \%)$ \\
\hline
\end{tabular}

${ }^{1} n=4$ participants smoked $<5$ CPD in the 7 days prior to the eligibility survey, but had missing data for their avg. CPD. For these participants, 2.5 CPD was assumed. 


\begin{tabular}{|l|l|}
\hline & $\begin{array}{l}\text { Mean } \pm \text { SD (range) } \\
\text { or } \mathbf{n}(\%)\end{array}$ \\
\hline $31-60$ minutes & $6(15.0 \%)$ \\
\hline $61+$ minutes & $4(10.0 \%)$ \\
\hline Alcohol-Use Severity (AUDIT-10 in Eligibility Survey) & $14.93 \pm 4.87(7-$ \\
& $24)$ \\
\hline $\begin{array}{l}{ }^{n} \mathrm{n}=4 \text { participants smoked < } 5 \text { CPD in the 7 days prior to the eligibility survey, but had missing data for } \\
\text { their avg. CPD. For these participants, 2.5 CPD was assumed. }\end{array}$ \\
\hline
\end{tabular}

\section{Intervention Characteristics}

The intervention included a combination of counseling and nicotine replacement therapy (NRT patch plus gum or lozenge) to help manage nicotine withdrawal. Overall the smoking and alcohol cessation intervention was perceived as acceptable by participants. Some participants described appreciating the opportunity to discuss their health, as much of the resource services offered by the shelter focused on addressing their homelessness. Participants who were randomized to the smoking and alcohol counseling arm (vs health education) mentioned the importance of their counselors' kind demeanor. Having a warm, friendly, and approachable attitude was key to participants' overall experience in the study.

"Yeah, as far as staff, I never felt like I was less than them just because of being in here at (the shelter). They always made me feel welcome. (Intervention group participant).

Counseling sessions, which became a part of participants' routine, were described as contributing to an increased sense of purpose. However, while most of the participants saw the benefits of the counseling sessions, some participants were resentful of having to participate in counseling sessions, and felt their counselor was nosy and intrusive. Some participants receiving the one time health education counseling session group believed they would have benefitted from going to the ongoing counseling sessions while others were relieved not to have to attend them. While a few participants believed they would have benefitted from more frequent sessions, for the most part participants were content with the amount of counseling sessions received.

"I liked it the whole session. I didn't just want the patches, to come and go. I needed the counseling, too" (Intervention group participant).

Overall, participants described that staff provided a comfortable and respectful environment, with the counseling providing a space for focus, reflection, motivation, and skill-building. Occupying one's time with other, non-smoking activities was a key strategy participants used when they had the urge to smoke or drink. Across the board, participants were educated about the consequences of smoking or drinking on the body and were able to reflect on the ways in which those behaviors were detrimental to their own 
health. The health consequences of tobacco or alcohol use strongly resonated with participants and the impact of this was present throughout participant responses.

In addition to counselling, participants were also offered Nicotine Replacement Therapy (NRT). NRT for the most part, was described as helping participants manage their cravings. NRT gum and lozenges were reported as being moderately successful in managing craving. However, some participants reported that the patch did not reduce the urge to smoke. Physiological cravings were reported to increased appetite and overeating, which subsequently led to weight gain and a fear of putting on weight.

\section{Outer Setting}

Participants described a range of needs outside of the intervention that impacted their experience of the study. A significant challenge related to the shelter environment was the perception of the ubiquitous use of cigarette smoking and alcohol abuse. In fact, some participants reported having started smoking for the first time since their stay at the shelter. Participants described experiencing frequent temptation and peer pressure to drink and smoke from other shelter residents in areas immediately around the shelter. Smoking and drinking were both described as very important in the social life for shelter residents.

"It's kind of hard, you're walking down the street and all of a sudden you're in a puff of smoke, you're like wait, I could use one of those" (Intervention group participant).

Getting to counselling sessions was convenient for participants who lived in the shelter; however, for participants who had moved to more stable housing during the study duration, returning to the shelter for appointments was a challenge. Additionally participants described challenges getting to appointments because of adverse weather, conflicts with work, and conflicts with doctor appointments.

The need to find housing, while having very limited financial resources, was another challenge for participants. Cutting back on cigarettes and problem drinking was reported as helping some participants alleviate the financial burden of smoking and/or drinking, cultivating feelings of accomplishment and pride in cutting back their consumption behaviors, and feeling better physically and emotionally.

Many participants described feeling that their personal strength and ability to focus on their goals was what led them to be a part of the study. Participants faced common challenges to smoking cessation, such as dealing with cravings and urges to smoke. Study participants were asked to set a goal of quitting smoking and drinking, however participants frequently identified that they often had their own goal of lessening smoking or drinking, rather than quitting. For many, smoking and drinking were described as habitually intertwined. Engagement in either habit was seen as a trigger spurring engagement in the other. Likewise, reduction or quitting of one, was also associated with the reduction or quitting of the other. Participants reported reductions in smoking or drinking as personal successes.

"I was doing like a couple packs a day, so for me to go from that to six cigarettes a day, that's like a miracle to me!" (Intervention group participant). 
Many participants described forming a bond with the PTQ2 staff and reliance on them for emotional support and encouragement. Many were also glad to have the opportunity to branch out and interact with different people.

\section{Inner Setting}

The shelter setting offered convenience for participants, however it also presented some challenges as it did not always feel very quiet or confidential to some. Additionally, while the shelters themselves were smoke-free and alcohol-free environments, the social pressure, direct or indirect, from fellow shelter residents was challenging. Despite this, participants described a range of motivators and expectations. Many were motivated to enroll for health reasons, including fear of future diagnoses such as cancer. Participants also described the belief that personal willpower was needed before being ready to engage with help and attempt to quit.

"First of all, change has to come from within; if you're not ready to change, you're not going to change. I got irons in every fire I can. My motto is I need all the help I can get!"'(Intervention group participant).

\section{Implementation Process}

Participants were mostly positive about their study participation. Participation was described as helping foster sober social time, positive feelings about contributing to the community, and a focused attitude to improve their situation.

"I think it's good. It made me feel like I had something to do or like I had a purpose. You know what I mean, not a purpose but it wasn't like the homeless" (Intervention group participant).

Across conditions, participants completed regular study outcome surveys at multiple time points throughout the trial. While a few participants viewed the survey with no particular value, the majority, including control arm participants, viewed this component of the study as meaningful, and helpful in monitoring and reducing smoking behaviors. Notably, nearly all participants were grateful and enthused by the financial incentives.

\section{Discussion}

In this paper, we have applied an implementation science framework, CFIR, to the analysis of the experience of participants in PTQ2 in order to enhance learning on how to best deliver smoking cessation and alcohol abstinence interventions to this at risk population. The outer setting in which the intervention was delivered presented unique challenges for study participants. In particular, the culture of alcohol use and cigarette smoking around the shelter environment presented a serious challenge. There may be a need to consider the impact of broader smoke free policies around shelters. These challenges have previously been reported in the literature. $(8,21,22)$ It has also been reported that the daily life challenges facing people experiencing homelessness can negatively impact smoking cessation; $(7,9,10)$ however, 
participants also described experiencing reduction or cessation as being a helpful strategy to help support broader goals surrounding attainment of permanent housing.

The inner setting of the intervention delivery also emerged as important. The inner setting of the shelters themselves offered a very convenient way to recruit smokers experiencing homelessness. However, this busy, chaotic setting, also proved challenging. As study participants moved away from the shelter, the convenience of the shelter setting transformed into a barrier. It was challenging for participants to return to the shelter, and doing so could expose individuals to pro-smoking and drinking behaviors. Stable housing has been associated with positive outcomes for smoking cessation,(23) and finding a way to move the intervention with participants when they move away from the shelter, may be helpful.

These was a discrepancy between the goals of the study, cessation, and the goals of individual participants, who felt that reduction was a worthwhile and significant achievement. This poses a challenge for the ways to best address smoking in this community and suggest the need for a broader consideration of the role of reduction in circumstances where there are significant barriers to overcome in the outer setting of the intervention. Additionally, participants reported that they gained value from the frequent surveys of their health, including of their cigarette and alcohol use, even for those participants who were in the control arm.

\section{Limitations}

This study has some limitations. The sample size is small and may not be representative. Additionally, study participants who felt they were successful in the study may have been more inclined to agree to participate in an interview, and may have overrepresented positive study experiences. Finally, this study specifically recruited smokers experiencing homelessness who also had alcohol use disorder, which may limit the generalizability of the findings beyond this particular group.

\section{Conclusion}

Overall, PTQ2 was well received by study participants, reinforcing the value of continuing to test and offer smoking cessation interventions for people experiencing homelessness. The CFIR framework(14) was useful in offering specific insights about the implementation context of the intervention. Participants described a discord in their personal goals of reduction compared with the study goals of complete abstinence, which may pose a challenge to the ways in which success is defined for people experiencing homelessness

\section{Declarations}

\section{Ethics approval and consent to participate}

Ethical approval was granted by the University of Minnesota Institutional Review Board. Written informed consent was provided by participants. 


\section{Consent for publication}

Not applicable.

\section{Availability of data and materials}

The datasets used and/or analyzed during the current study are available from the corresponding author on reasonable request.

\section{Competing interests}

The authors declare that they have no competing interests.

\section{Funding}

This work was supported by the National Heart, Lung, and Blood Institute under Grant number . Effort for the co-author (SX) was supported by the National Institutes of Health's National Center for Advancing Translational Sciences grant TL1R002493. The content is solely the responsibility of the author and does not necessarily represent the official views of the National Institutes of Health's National Center for Advancing Translational Sciences.

\section{Authors' contributions}

All drafted and approved the manuscript. In addition RP, SX, AK conceptualization, data analysis and interpretation. CSA, AJ, SAE, XL, NC, JT, SS and KO conceptualization and interpretation.

\section{Acknowledgements}

Not applicable.

\section{References}

1. Tsai J. Lifetime and 1-year prevalence of homelessness in the US population: results from the National Epidemiologic Survey on Alcohol and Related Conditions-III. J Public Health (Bangkok). 2017 Mar;40(1):1-10. doi:10.1093/pubmed/fdx034

2. Hwang, S. Homelessness and health. Can Med Assoc Journal. 2001. 164 (2) 229-233.

3. Baggett TP, Lebrun-Harris LA, Rigotti NA. Homelessness, cigarette smoking and desire to quit: results from a US national study. Addiction. 2013 Nov;108(11):2009-18. doi.wiley.com/10.1111/add.12292

4. Reitsma M, Fullman N, Ng M, Salama J, Abajobir A, Abate, K, et al. Smoking prevalence and attributable disease burden in 195 countries and territories, 1990-2015: a systematic analysis from the Global Burden of Disease Study 2015. Lancet. 2017 May;389(10082):1885-1906. doi:10.1016/S0140-6736(17)30819-X 
5. Baggett TP, Chang Y, Singer DE, Porneala BC, Gaeta JM, O'Connell JJ, et al. Tobacco-, alcohol-, and drug-attributable deaths and their contribution to mortality disparities in a cohort of homeless adults in Boston. Am J Public Health. 2015 Jun;105(6):1189-97. doi:10.2105/AJPH.2014.302248

6. Mullins L, O'Hanlon C, Shadel, W, Tucker, J. qualitative study of smoking cessation experiences and perceptions among homeless young adults. Journal of Social Distress and Homlessness. 2017. 27 (1) 1-8.

7. Bonevski B, Baker A, Twyman L, Paul C, Bryant J. Addressing smoking and other health risk behaviours using a novel telephone-delivered intervention for homeless people: A proof-of-concept study. Drug Alcohol Rev. 2012 Jul;31(5):709-13. doi: 10.1111/j.1465-3362.2012.00438.x

8. Baggett TP, Chang Y, Yaqubi A, McGlave C, Higgins ST, Rigotti NA. Financial incentives for smoking abstinence in homeless smokers: A pilot randomized controlled trial. Nicotine Tob Res. 2018. 20(12):1442-1450.

9. Glenn N, Lapalme J, McCready G, Frohilich KL. Young adults' experiences of neighbourhood smoking-related norms and practices: A qualitative study exploring place-based social inequalities in smoking. Soc Sci Med. 2017 Sep 1;189:17-24. doi: 0.1016/J.SOCSCIMED.2017.07.021

10. Okuyemi KS, Goldade K, Whembolua G-L, Thomas JL, Eischen S, Sewali B, et al. Motivational interviewing to enhance nicotine patch treatment for smoking cessation among homeless smokers: a randomized controlled trial. Addiction. 2013 Jun;108(6):1136-44. doi: 10.1111/add.12140

11. Pratt R, Pernat C, Kerandi L, Kmiecik A, Strobel-Ayres C, Joseph A, et al. "It's a hard thing to manage when you're homeless": the impact of the social environment on smoking cessation for smokers experiencing homelessness. BMC Public Health. 2019. 19, 635

12. Maddox S, Segan C. Underestimation of homeless clients' interest in quitting smoking: a case for routine tobacco assessment. Heal Promot J Aust. 2017 Aug 30;28(2):160-4. doi: 10.1071/HE15102

13. Nilsen P. Making sense of implementation theories, models and frameworks. Implement Sci. 2015. (10):53.

14. Damschroder LJ, Aron DC, Keith RE, Kirsh SR, Alexander JA, Lowery JC. Fostering implementation of health services research findings into practice: a consolidated framework for advancing implementation science. Implement Sci. 2009;4(1):50.

15. Kirk MA, Kelley C, Yankey N, Birken SA, Abadie B, Damschroder L. A systematic review of the use of the Consolidated Framework for Implementation Research. Implement Sci [Internet]. 2015;11(1):72.

16. Damschroder L, Hall C, Gillon L, Reardon C, Kelley C, Sparks J, et al. The Consolidated Framework for Implementation Research (CFIR): progress to date, tools and resources, and plans for the future. Implement Sci [Internet]. 2015. 10(S1):A12.

17. Davies S, Burton CR, Williams L, Tinkler A. Brief smoking cessation in acute Welsh hospitals: a realist approach. Health Promot Int. 2019. 35(2):244-254

18. VanDevanter N, Kumar P, Nguyen N, Nguyen L, Nguyen T, Stillman F, et al. Application of the Consolidated Framework for Implementation Research to assess factors that may influence 
implementation of tobacco use treatment guidelines in the Viet Nam public health care delivery system. Implement Sci [Internet]. 2017. 12(1):27.

19. Sorensen J, Kosten, T. Developing the tools of implementation science in substance use disorders treatment: Applications of the consolidated framework for implementation research. Psychol Addict Behav. 2011. 25(2):262-268.

20. Hagedorn HJ, Wisdom JP, Gerould H, Pinsker E, Brown R, Dawes M, et al. Implementing alcohol use disorder pharmacotherapy in primary care settings: a qualitative analysis of provider-identified barriers and impact on implementation outcomes. Addict Sci Clin Pract. 2019 Jul 10;14(1):24.

21. Stewart HC, Stevenson TN, Bruce JS, Greenberg B, Chamberlain LJ. Attitudes Toward Smoking Cessation Among Sheltered Homeless Parents. J Community Health. 2015: 40(6):1140-8.

22. Vijayaraghavan M, Hurst S, Pierce JP. A qualitative examination of smoke-free policies and electronic cigarettes among sheltered homeless adults. Am J Heal Promot. 2017 May 11;31(3):243-50. doi: 10.4278/ajhp.150318-QUAL-781

23. Businelle MS, Cuate EL, Kesh A, Poonawalla IB, Kendzor DE. Comparing homeless smokers to economically disadvantaged domiciled smokers. Am J Public Health. 2013 Dec;103(S2):S218-20. doi: 10.2105/AJPH.2013.301336

24. Schanzer B, Dominguez B, Shrout PE, Caton CLM. Homelessness, health status, and health care use. Am J Public Health. 2007: 97(3):464-9. Available from: http://ajph.aphapublications.org/doi/10.2105/AJPH.2005.076190

25. Porter M, Harvey J, Gavin J, Carpenter M, Cummings K, Pope C, et al. A qualitative study to assess factors supporting tobacco use in a homeless population. AlMS Med Sci. 2017 Feb 21;4(1):83-98. doi: 10.3934/medsci.2017.1.83

26. Boland VC, Mattick RP, McRobbie H, Siahpush M, Courtney RJ. "I'm not strong enough; I'm not good enough. I can't do this, I'm failing": a qualitative study of low-socioeconomic status smokers' experiences with accessing cessation support and the role for alternative technology-based support. Int J Equity Health. 2017;16(1):196. Available from: https://equityhealthj.biomedcentral.com/articles/10.1186/s12939-017-0689-5.

27. Baggett TP, Rigotti NA. Cigarette Smoking and Advice to Quit in a National Sample of Homeless Adults. Am J Prev Med. 2010:39(2):164-72.

28. Reitzel LR, Nguyen N, Eischen S, Thomas J, Okuyemi KS. Is smoking cessation associated with worse comorbid substance use outcomes among homeless adults? Addiction. 2014:109(12):2098104.

29. Torchalla I, Strehlau V, Okoli CTC, Li K, Schuetz C, Krausz M. Smoking and Predictors of Nicotine Dependence in a Homeless Population. Nicotine Tob Res. 2011:13(10):934-42. doi/10.1093/ntr/ntr101

30. Kalman D, Kim S, DiGirolamo G, Smelson, D, Ziedonis, D. Addressing tobacco use disorder in smokers in early remission from alcohol dependence: the case for integrating smoking cessation services in substance use. Clinical Psychology Review. 2010: 30(1): 12-24. 
31. Gulliver S, Kamholz B, Helstrom, A. Smoking cessation and alcohol abstinence: What do the data tell us? Alcohol Research and Health. 2006. 29(3): 208-212.

32. Kodl M, Fu S, Joseph, A. Tobacco cessation treatment for alcohol-dependent smokers: when is the best time? Alcohol Research and Health. 2006:29(3): 203-207.

33. Burling T, Burling, A, Latini, D. A controlled smoking cessation trial for substance-dependent inpatients. psycnet. J Consult Clin Psychol. 2001. 69(2) 295-304.

34. Ojo-Fati O, Joseph AM, Ig-Izevbekhai J, Thomas JL, Everson-Rose SA, Pratt R, et al. Practical issues regarding implementing a randomized clinical trial in a homeless population: strategies and lessons learned. Trials [Internet]. 2017 Dec 5 [cited 2018 Feb 26];18(1):305. Available from: http://trialsjournal.biomedcentral.com/articles/10.1186/s13063-017-2046-9

35. Ojo-Fati O, John F, Thomas J, Joseph AM, Raymond NC, Cooney NL, et al. Integrating smoking cessation and alcohol use treatment in homeless populations: study protocol for a randomized controlled trial.Trials. 2015:16:385.

36. Ltd. QSRIP. NVivo qualitative data analysis Software. Version 10. QSR International Pty Ltd; 2012.

37. Charmaz K. Constructing grounded theory. 2nd ed. 2014. London: Sage Publications.

38. Charmaz K. Grounded theory. In: Smith J, Harre R, Van Langenhove L, editors. Rethinking methods in psychology. London: Sage Publications; 1995. p. 27-49.

39. Bohn MJ, Babor TF, Kranzler HR. The alcohol use disorders identification test (AUDIT): validation of a screening instrument for use in medical settings. J Stud Alcohol. 1995 Jul;56(4):423-32. doi/10.15288/jsa.1995.56.423

\section{Supplementary Files}

This is a list of supplementary files associated with this preprint. Click to download.

- TIDieRChecklistWord.docx 